\title{
Dissect the Heterogeneity of Longitudinal Cognitive Declines with Risk Factors and Functional Outcomes in Bipolar Disorder
}

\section{Wen Yin Chen}

Taipei City Hospital Songde Branch https://orcid.org/0000-0001-8626-6472

\section{Ming-Chyi Huang}

Taipei City Hospital Songde Branch

\section{Ya-Chin Lee}

National Taiwan University Institute of Epidemiology and Preventive Medicine

\section{Chiao-Erh Chang}

National Taiwan University Institute of Preventive Medicine: National Taiwan University Institute of

Epidemiology and Preventive Medicine

\section{Shih-Ku Lin}

Taipei city hospital, songde branch

\section{Chih-Chiang Chiu}

Taipei city hospital, songde branch

\section{Hsing-Cheng Liu}

Taipei city hospital, songde branch

\section{Chian-Jue Kuo}

Taipei city hospital, songde branch

\section{Po-Yu Chen}

Taipei city hospital, songde branch

Po-Hsiu Kuo ( $\nabla$ phkuo@ntu.edu.tw)

College of Public Health, National Taiwan University https://orcid.org/0000-0003-0365-3587

\section{Research}

Keywords: BAC-A, bipolar disorder, function assessment, heterogeneity, longitudinal

Posted Date: October 30th, 2020

DOI: https://doi.org/10.21203/rs.3.rs-97680/v1

License: (c) (1) This work is licensed under a Creative Commons Attribution 4.0 International License. Read Full License 


\section{Abstract}

\section{Background:}

We characterized the heterogeneity and risk factors of cognitive decline in bipolar disorder (BD), and their magnitude of associations with subjective daily functions.

\section{Methods:}

Recruited BD patients ( $N=127)$ were followed for an average of 6.5 years. Baseline intelligence quotient was measured, and Brief Assessment of Cognition in Affective Disorders (BAC-A) was used to assess cognition at follow-up. We evaluated current functions with World Health Organization Disability Assessment Schedule 2.0. Clinical and sociodemographic factors were compared between BD patients with and without longitudinal cognitive decline and revealed the independent risk factors through multiple regression analysis. Correlations between specific cognitive domains and functional outcomes were examined in subgroups of BD.

\section{Results:}

Overall, $32.3 \%$ of BD patients showed longitudinal cognitive decline. In cognitive decline group, all cognitive domains of BAC-A were significantly worsened. Baseline independent risk factors for cognitive decline were older age, body mass index $>25$, and manic episode density during the disease course. After the age of 40 years, a steeper cognitive change was observed in the cognitive decline group. Housekeeping dysfunction was the most sensitive function related to diverse cognitive impairment in cognitive decline group. The correlations between cognitive domains and functional outcomes differed between patients with and without cognitive decline.

\section{Conclusions:}

This study characterized cognitive heterogeneity longitudinally in BD, as the foundation to facilitate future genetic and biological studies for BD subtypes. Manic episode density and overweight are important risk factors for cognitive decline; therefore, we suggested further interventions should target on relapse prevention and controlling body weight.

\section{Introduction}

Bipolar disorder (BD), a recurrent chronic disorder, is characterized by episodes of mania and depression interspaced by euthymia, and it affects different aspects of daily living (Goldberg et al. 1995). BD is also associated with cognitive deficits in a number of domains, including executive function, attention, and memory, which may persist in patients in remission (Bora et al. 2015). For example, Robinson et al. reported that euthymic patients with BD showed cognitive impairments compared with healthy controls, particularly in executive function and verbal learning (Robinson et al. 2006). A meta-analysis using individual patient data showed significant cognitive impairments in BD after controlling for age, 
educational years, gender, residual mood symptoms, and medications (Bourne et al. 2013). Cognitive deficits have substantial negative impacts on social functioning and are responsible for poor interepisode recovery or poor quality of life in a high proportion of patients (Atre-Vaidya et al. 1998, Duarte et al. 2016, Cipriani et al. 2017), suggesting the importance of studying cognitive functions in BD to enhance future clinical care and prognosis outcomes.

Previous cross-sectional studies have examined some clinical factors with cognitive deficits in BD. Although inconsistent, some studies have reported the correlations of cognitive impairments with illness severity. Number of manic episodes and number of total episodes were positively correlated with impairments in set-shifting and processing speed (Bourne et al. 2013). However, it is difficult to infer the causal link between cognitive deficits and clinical features due to the cross-sectional nature of these studies. One early study adopted a first-episode design to compare first-episode BD, schizophrenia, and healthy controls, and found that cognitive deficits are evident right from the first mood episode (Bora 2015). During the disease course, cognitive impairment in BD patients then varies, with some studies suggesting that cognitive deficits stabilize over time (Mur et al. 2008, Gildengers et al. 2013, Samame et al. 2014), whereas others have shown a pattern of progressive deterioration (Moorhead et al. 2007, Gildengers et al. 2009) and even increased risk of dementia in later life (Diniz et al. 2017). It is believed that heterogeneity is widely observed among BD patients in this regard, in terms of the impaired cognitive domains, longitudinal cognition stability, and the speed of deteriorative progression. Longitudinal study design is preferable to explore cognitive declines over the course of bipolar illness, though it was relatively scarce in the literature.

So far, the heterogeneity of longitudinal cognitive declines in BD patients is not well understood. First, insufficient data were available to investigate all cognitive domains in few longitudinal studies, and findings on deficits within different cognitive domains were inconsistent. Whether these are discrete areas of impairment or reflect an underlying single, more basic cognitive abnormality is as yet unclear. Second, previous longitudinal studies often consisted of small sample sizes and different follow-up periods, resulted in various findings. For example, the latest synthesis of longitudinal evidence suggests that the cognitive path of individuals with BD may be set early on and may not deteriorate over time (Szmulewicz et al. 2020). However, another report with a much longer follow-up period showed significant test-retest differences in executive measures revealing decline (Torrent et al. 2012), which implied the cognitive instability over longer disease course may exist. Third, the differences in medication variables or mood scales between assessment times could not be well controlled in every study and may have influenced the results. In addition, we should consider the effect of repeated testing, wherein the true decline of cognitive functioning would be partially masked by learning effects.

In the present study, we considered that both the clinical course and cognitive decline may be heterogeneous among BD patients, and thus may partially account for the inconsistency of findings in previous reviews. The majority of cross-sectional studies have suggested that $38-40 \%$ of patients with BD have no neurocognitive deficits, and $20-30 \%$ had obvious cognitive deficits (Martino et al. 2008, Bora et al. 2016, Volkert et al. 2016). The lack of longitudinal assessment of cognitive performance in BD 
hinders to explore the heterogeneity in cognitive deficits. Therefore, we aimed to evaluate the cognitive changes in $\mathrm{BD}$ patients over time by looking at age hinge points of cognitive declines, to characterize heterogeneous cognitive profiles among BD patients, and to examine the risk factors for cognitive decline, as whether different cognitive changes impact daily functions in patients with and without cognitive declines differently.

\section{Methods}

\section{Participants}

Participants who were diagnosed with BD according to Diagnostic and Statistical Manual of Mental Disorders, 4th Edition, Text Revision (DSM-IV-TR) and referred by board-certified psychiatrists were enrolled from Taipei City Hospital, Songde branch. All participants drawn from who had a history of prior baseline intelligence quotient (IQ) testing during euthymic state in the past medical record. In addition, they were more than 20 years of age, and those without: a) known substance use disorder (except nicotine use disorder); b) any disorder with known neurological symptoms or complications such as brain injury or stroke; $c$ ) a diagnosis of intellectual disabilities; or d) inability to complete the standard clinical assessment or providing informed consent. Individuals were also required to be euthymic at the time of study entry for cognitive assessment. Mood symptoms were obtained through clinician-administered measures of the 17-item Hamilton Rating Scale for Depression (HRSD-17) and Young Mania Rating Scale (YMRS). For euthymic state, we defined HRSD and YMRS scores $\leq 8$ within 7 days before assessment.

\section{Measurements}

\section{Demographic data, clinical course, and baseline IQ}

Patients' demographic and clinical course data were collected from medical records and interviews by psychiatrists, if required. The clinical characteristics of participants included the number of affective episodes, number of episodes with psychotic features, number of hospitalizations, age at illness onset, maximum length of free intervals, and number of suicide attempts. Considering the duration of illness varied across individuals, we also calculated episode density by dividing number of total episodes and manic episodes by duration of illness as the total episode density and manic episode density, respectively. Through chart review, we recorded their physical comorbidities, anthropometry, and baseline IQ. For this recorded baseline IQ, it was measured by a licensed psychologist using the Wechsler Adult Intelligence Scale (WAIS-III or WAIS-IV) in BD euthymic state for the clinical practice as the attending physicians start beginning for follow up the patients. Psychopharmacological treatments used at the time of study assessment were also transformed to defined daily dose (DDD) and then recorded.

\section{Cognitive measurements}


Patients were assessed using the Brief Assessment of Cognition in Affective Disorders (BAC-A) during their euthymic state for at least 1-year interval compared with their baseline IQ measurements to ensure the longitudinal change from the possible learning effects. BAC-A has extensively been used as a rapid and reliable measure of cognitive assessment in a range of clinically affective patients (Cholet et al. 2014). It provides not only measure of affective memory and emotional inhibition (Affective Processing Tests [APTs]), but also includes six traditional neurocognitive domains, namely, working memory, motor speed, verbal fluency, attention and processing speed, verbal memory, and executive function (Bauer et al. 2015). The criterion and construct validity of each test for cognitive impairment as well as the sensitivity of these tests to changes in cognition have been demonstrated in the scientific literature, and each test has also been shown to be valid for use in different cultures and language groups (Lee et al. 2018).

\section{Functional assessment}

Trained interviewer administered the World Health Organization Disability Assessment Schedule 2.0 (WHODAS 2.0) for multidimensional assessment of function in the participants. WHODAS 2.0 is a practical, generic assessment instrument that can measure health and disability at a population level or in clinical practice. It evaluates subjective function in six domains: Domain 1: cognition- understanding and communicating; Domain 2: Mobility- moving and getting around; Domain 3: Self-care- attending to one's hygiene, dressing, eating, and staying alone; Domain 4: Getting along- interacting with other people; Domain 5: Life activities- domestic responsibilities, leisure, work, and school; and Domain 6:

Participation- joining in community activities and participating in society.(Ustun et al. 2010) The raw score is transformed into item response theory (IRT) scoring (0-100), with higher scores indicating increasing severity of subjective impairment (Ustun et al. 2010).

\section{Statistical analysis}

We defined cognitive heterogeneity in BD longitudinally and dichotomously. Patients who fulfilled one of the following criteria were included in the cognitive decline group: First, if their baseline IQ scores were less than the estimated norm based on their previous educational and occupational history assessed by a licensed psychologist. Second, if their baseline IQ showed a difference of more than 2 standard deviation (SD) compared with the current composite z-score of the BAC-A. Those participants without above conditions will regard as group without cognitive decline.

We compared demographic and clinical characteristics between BD patients with and without cognitive decline using the chi-square and Student's $t$ test for categorical and continuous variables, respectively. The differences in BAC-A and functional outcomes were also examined. To assess independent risk factors, potential confounding variables were included in multivariable logistic regression analysis. Spearman's correlations between specific cognitive and functional domains were demonstrated in each subgroup. Moreover, multivariate adaptive regression spline (MARS) (Vanegas et al. 2017) and scatterplot with Loess curve were used to detect hinges or inflection points to characterize the timing and nature of slope changes in the BAC-A composite scores of separate cognitive groups. MARS analysis was 
conducted using the earth package in R (Milborrow 2011); all other analyses were conducted using SAS (version 9.4; SAS Institute Inc.; Cary, NC, USA). Significance was set at $p<0.05$.

\section{Results}

\section{Patient characteristics}

Of the total $127 \mathrm{BD}$ patients, $67.7 \%(\mathrm{~N}=86)$ and $32.3 \%(\mathrm{~N}=41)$ were without and with longitudinal cognitive decline, respectively (Table 1). The mean period from baseline IQ to current BAC-A assessment in BD patients was 6.5 years, which was no difference between the two subgroups. Compared with patients without cognitive decline, those with cognitive decline experienced more mood episodes, especially manic episodes, more number of admissions, and higher hospitalization duration, and they showed higher baseline body mass index (BMI), as assessed at their index admission (the earliest record from the medical chart system). No significant differences were observed in variables such as physical comorbidities, duration of illness, age of onset, or baseline IQ measurement between the two groups. In addition, there was also no difference in the two groups for their current DDD of psychoactive agents used, which implied that medication was not the cofounding factor in our further analysis. 
Table 1

Sociodemographic and clinical characteristics of BD patients with and without cognitive decline

\begin{tabular}{|c|c|c|c|}
\hline & $\begin{array}{l}\text { Without Cognitive } \\
\text { decline } \\
(\mathrm{N}=86)\end{array}$ & $\begin{array}{l}\text { With Cognitive } \\
\text { decline } \\
(\mathrm{N}=41)\end{array}$ & P-value \\
\hline Gender Male, n (\%) & $52(60.47)$ & $22(53.66)$ & 0.467 \\
\hline $\begin{array}{l}\text { Marriage: married or lived together, } \mathrm{n} \\
(\%)\end{array}$ & $34(39.53)$ & $10(24.39)$ & 0.094 \\
\hline Job: regular job, n (\%) & $25(29.07)$ & 6 (14.63) & 0.077 \\
\hline With family psychiatry history, n (\%) & $37(44.58)$ & $15(36.59)$ & 0.396 \\
\hline Smoking, n (\%) & $14(16.67)$ & 7 (17.07) & 0.648 \\
\hline Alcohol use habit, n (\%) & $10(11.77)$ & $7(17.08)$ & 0.418 \\
\hline Current physical comorbidity, n (\%) & $16(19.05)$ & $11(26.83)$ & 0.321 \\
\hline Age (yr) & $48.30 \pm 12.32$ & $51.71 \pm 14.43$ & 0.171 \\
\hline Onset of age (yr) & $26.48 \pm 10.67$ & $29.03 \pm 13.99$ & 0.311 \\
\hline Education years & $13.33 \pm 2.61$ & $11.83 \pm 4.67$ & 0.060 \\
\hline Baseline full IQ & $89.81 \pm 16.71$ & $88.07 \pm 17.77$ & 0.730 \\
\hline performance IQ & $86.76 \pm 19.59$ & $80.68 \pm 17.49$ & 0.258 \\
\hline verbal IQ & $93.67 \pm 15.78$ & $96.57 \pm 17.71$ & 0.555 \\
\hline Follow up duration (yr) & $5.38 \pm 4.42$ & $7.31 \pm 7.20$ & 0.248 \\
\hline Duration of illness (yr) & $21.93 \pm 10.64$ & $22.78 \pm 11.12$ & 0.683 \\
\hline Number of total episodes & $7.64 \pm 4.92$ & $10.15 \pm 6.62$ & $0.037 *$ \\
\hline Number of total manic episodes & $5.35 \pm 4.28$ & $7.63 \pm 6.13$ & $0.038^{*}$ \\
\hline Number of total depressive episode & $2.17 \pm 2.20$ & $2.18 \pm 2.64$ & 0.999 \\
\hline Number of mixed episodes & $0.10 \pm 0.67$ & $0.35 \pm 0.77$ & 0.070 \\
\hline Number of psychotic episodes & $3.02 \pm 3.56$ & $5.22 \pm 6.64$ & 0.052 \\
\hline Maximum of free interval (month) & $84.97 \pm 66.16$ & $78.18 \pm 62.81$ & 0.587 \\
\hline Number of suicide attempt & $0.81 \pm 1.63$ & $1.17 \pm 1.90$ & 0.275 \\
\hline \multicolumn{4}{|l|}{$* p<0.05$} \\
\hline IQ, intelligence quotient; DDD, defined & ly dose & & \\
\hline
\end{tabular}




\begin{tabular}{|llll|}
\hline Days of hospitalization & $199.40 \pm 210.10$ & $317.00 \pm 261.10$ & $0.008^{*}$ \\
\hline Number of admission & $5.34 \pm 5.61$ & $8.20 \pm 8.08$ & $0.048^{*}$ \\
\hline Baseline body mass index, BMI & $22.65 \pm 3.60$ & $25.65 \pm 4.68$ & $0.0002^{\star}$ \\
\hline Current psychoactive agents (DDD) & & & \\
\hline First-generation antipsychotics & $0.06 \pm 0.18$ & $0.12 \pm 0.38$ & 0.391 \\
\hline Second-generation antipsychotics & $3.76 \pm 5.05$ & $5.12 \pm 6.39$ & 0.197 \\
\hline Mood stabilizers & $0.52 \pm 0.35$ & $0.61 \pm 0.43$ & 0.216 \\
\hline Antidepressants & $0.03 \pm 0.19$ & $0.01 \pm 0.04$ & 0.262 \\
\hline Benzodiazepines & $0.49 \pm 0.74$ & $0.54 \pm 0.66$ & 0.730 \\
\hline *p<0.05 & & \\
\hline IQ, intelligence quotient; DDD, defined daily dose & & \\
\hline
\end{tabular}

\section{Functional outcomes and BAC-A in BD patients with cognitive decline}

No significant differences were observed in current functional outcomes assessed using WHODAS 2.0 between BD patients with or without cognitive decline. Table 2 shows the IRT scoring of the groups; a higher score indicated more severity of subjective impairment, that is, greater disability. We noted that common functional impairments in the chronic phase of BD were observed in both groups, regardless of presence of cognitive decline. In patients with cognitive decline, the functional domain with the highest score was job and learning (IRT score 86.67), implying that it was the most severely impaired domain compared with the norm (around last 1\% in the population percentile) (Ustun et al. 2010). Even for the most preserved functional domain (self-care), patients showed impairment that was the last $30 \%$ in the population percentile when compared with norm data (Ustun et al. 2010). 
Table 2

Mood status and functional assessment using WHODAS 2.0 at study entry in BD patients with and without cognitive decline

\begin{tabular}{|llll|}
\hline WHODAS2.0 & $\begin{array}{l}\text { Without } \\
\text { Cognitive decline } \\
(\mathbf{N}=\mathbf{8 6})\end{array}$ & $\begin{array}{l}\text { With } \\
\text { Cognitive decline } \\
(\mathbf{N}=\mathbf{4 1})\end{array}$ & \\
\hline Item-response-theory based score & & & \\
\hline D1 cognition & $23.75 \pm 17.02$ & $31.32 \pm 18.55$ & 0.172 \\
\hline D2 walk & $12.76 \pm 18.19$ & $20.42 \pm 29.16$ & 0.323 \\
\hline D3 self-care & $8.96 \pm 12.46$ & $14.21 \pm 19.53$ & 0.314 \\
\hline D4 along with others & $34.68 \pm 22.99$ & $29.95 \pm 15.57$ & 0.445 \\
\hline D5-1 housekeeping & $32.48 \pm 29.36$ & $25.26 \pm 30.44$ & 0.431 \\
\hline D5-2 job \& learn & $64.10 \pm 44.25$ & $86.67 \pm 35.19$ & 0.114 \\
\hline D6 social & $34.08 \pm 23.85$ & $25.37 \pm 17.17$ & 0.185 \\
\hline Total score & $28.72 \pm 17.44$ & $31.63 \pm 16.24$ & 0.575 \\
\hline HDS score & $2.36 \pm 2.03$ & $2.85 \pm 1.97$ & 0.199 \\
\hline YMRS score & $2.67 \pm 2.28$ & $2.73 \pm 2.33$ & 0.896 \\
\hline HDS, Hamilton depression scale; YMRS, Young mania rating scale & \\
\hline
\end{tabular}

Table 3 shows the cognitive profiles for BD patients with or without cognitive decline. Each patient's performance in individual tests was compared with an age- and gender-matched norm to calculate the zscore for six traditional neurocognitive domains. APTs were applied in addition to the six BAC-A subtests with the indexes of Affective Interference Test (AIT), Emotion Inhibition Test (EIT), Delayed Recognition (DR), and Emotion Inhibition Index (EII) (Chen et al. 2019). As observed in BAC-A assessment, the cognitive decline group showed significant worsening of all traditional neurocognitive domains. Moreover, APT indexes indicated the different emotional inhibitions between the groups. 
Table 3

Cognitive profiles at follow-up in BD patients with and without cognitive decline

\begin{tabular}{|c|c|c|c|}
\hline & $\begin{array}{l}\text { Without } \\
\text { Cognitive decline } \\
(\mathrm{N}=86)\end{array}$ & $\begin{array}{l}\text { With } \\
\text { Cognitive decline } \\
(\mathrm{N}=41)\end{array}$ & P-value \\
\hline BAC-A composite score & $-0.47 \pm 7.03$ & $-3.28 \pm 1.66$ & $<0.001^{*}$ \\
\hline Verbal memory & $-0.57 \pm 4.31$ & $-2.30 \pm 1.04$ & $<0.001^{*}$ \\
\hline Motor speed & $0.71 \pm 7.82$ & $-1.46 \pm 1.18$ & $0.014^{\star}$ \\
\hline Working memory & $-0.22 \pm 2.57$ & $-2.02 \pm 1.80$ & $<.0001^{*}$ \\
\hline Verbal fluency & $-1.36 \pm 3.64$ & $-2.33 \pm 0.72$ & $0.020^{*}$ \\
\hline Attention and processing speed & $-0.37 \pm 5.52$ & $-2.62 \pm 1.37$ & $<0.001 *$ \\
\hline Executive function & $-0.13 \pm 1.51$ & $-1.55 \pm 2.02$ & $<0.001^{*}$ \\
\hline \multicolumn{4}{|l|}{ Affective Processing Tests } \\
\hline AIT: total non-affective words & $13.44 \pm 4.44$ & $11.12 \pm 4.08$ & $0.006^{*}$ \\
\hline AIT: total affective words & $11.97 \pm 6.36$ & $7.54 \pm 4.56$ & $<.0001^{*}$ \\
\hline AIT: cued non-affective words & $3.84 \pm 2.04$ & $2.76 \pm 1.84$ & $0.005^{\star}$ \\
\hline AIT: cued affective words & $5.43 \pm 1.91$ & $4.17 \pm 1.70$ & $0.001^{\star}$ \\
\hline DR: correct non-affective words & $17.62 \pm 2.69$ & $16.73 \pm 2.71$ & 0.087 \\
\hline DR: correct affective words & $17.33 \pm 2.47$ & $15.73 \pm 2.98$ & $0.002^{*}$ \\
\hline DR: non-affective false alarms & $2.47 \pm 3.09$ & $3.27 \pm 2.71$ & 0.158 \\
\hline DR: affective false alarms & $3.09 \pm 2.80$ & $4.27 \pm 2.98$ & $0.032^{*}$ \\
\hline EIT: color naming score & $42.98 \pm 14.80$ & $31.02 \pm 12.88$ & $<.0001^{\star}$ \\
\hline EIT: neutral color word score & $36.28 \pm 11.02$ & $27.29 \pm 9.75$ & $<.0001^{\star}$ \\
\hline EIT: affective color word score & $35.60 \pm 10.73$ & $27.44 \pm 9.74$ & $<.0001^{\star}$ \\
\hline EIT: neutral word score & $40.98 \pm 11.05$ & $30.41 \pm 13.87$ & $<.0001 *$ \\
\hline Ell (emotion inhibition index) & $-83.28 \pm 21.74$ & $-61.59 \pm 25.25$ & $<.0001 *$ \\
\hline \multicolumn{4}{|l|}{${ }^{\star} p<0.05$} \\
\hline \multicolumn{4}{|c|}{ AIT, affective interference test; DR, delayed recognition; EIT, emotion inhibition test } \\
\hline
\end{tabular}


We used univariate and multivariable logistic regression to analyze the independent risk factors for cognitive decline in $\mathrm{BD}$, and results are displayed in Table 4. In multivariable logistic regression models, we selected manic episode density into the model and left out the total episode density to avoid the multicollinearity problem (correlation coefficient between total and manic episode density was high, equals to 0.89 ). The final model showed that age $>65$ years (odds ratio [OR] 6.02, 95\% $\mathrm{Cl} 1.37-26.51, p=$ 0.018 ), $\mathrm{BMI}>25$ at baseline (OR $3.01,95 \% \mathrm{Cl} 1.25-7.23, p=0.014$ ), and higher manic episode density (OR $12.18,95 \% \mathrm{Cl} 1.56-95.03, p=0.017$ ) were independent risk factors for cognitive decline after adjusted for age, gender, and years of education.

Table 4

Risk factors for cognitive decline in $\mathrm{BD}$ using univariate and multiple logistic regression analyses

\begin{tabular}{|c|c|c|c|c|c|c|}
\hline & \multicolumn{3}{|c|}{ Univariate } & \multicolumn{3}{|c|}{ Multivariable } \\
\hline & OR & $(95 \% \mathrm{Cl})$ & $\mathrm{p}$-value & OR & $(95 \% \mathrm{Cl})$ & p-value \\
\hline \multicolumn{7}{|l|}{ Age } \\
\hline$\leqq 65 y / 0$ & 1.00 & & & 1.00 & & \\
\hline$>65 y / 0$ & 5.77 & $(1.66-20.06)$ & $0.006 *$ & 6.02 & $(1.37-26.51)$ & $0.018^{*}$ \\
\hline \multicolumn{7}{|l|}{ Gender } \\
\hline Female & 1.00 & & & 1.00 & & \\
\hline Male & 1.32 & $(0.62-2.80)$ & 0.468 & 1.72 & $(0.73-4.05)$ & 0.214 \\
\hline \multicolumn{7}{|l|}{ Baseline BMI } \\
\hline$\leqq 25$ & 1.00 & & & 1.00 & & \\
\hline$>25$ & 3.60 & $(1.61-8.03)$ & $0.002^{\star}$ & 3.01 & $(1.25-7.23)$ & $0.014^{\star}$ \\
\hline Education years & 0.88 & $(0.79-0.99)$ & $0.027^{*}$ & 0.92 & $(0.81-1.05)$ & 0.210 \\
\hline Number of admissions & 1.07 & $(1.01-1.13)$ & $0.035^{\star}$ & & & \\
\hline Number of total episodes & 1.09 & $(1.01-1.17)$ & $0.030 *$ & & & \\
\hline Number of manic episodes & 1.10 & $(1.01-1.19)$ & $0.029 *$ & & & \\
\hline Total episode density & 4.22 & $(1.08-16.56)$ & $0.039 *$ & & & \\
\hline Manic episode density & 7.99 & $(1.49-42.82)$ & $0.015^{\star}$ & 12.18 & $(1.56-95.03)$ & $0.017 *$ \\
\hline \multicolumn{7}{|l|}{${ }^{\star} p<0.05$} \\
\hline $\mathrm{BMI}$, body mass index & & & & & & \\
\hline
\end{tabular}

Figure 1 depicts a steeper decline in BAC-A composite score in patients with cognitive decline compared with those without cognitive decline. In addition, MARS yielded a hinge at the age of 40 years for the 
cognitive decline group, and their BAC-A composite score steeply worsened by 0.1009 SD per year after this age.

\section{Correlation between cognitive and functional domains}

Figure 2 demonstrated correlations between cognitive and functional domains in patients with and without cognitive decline. The cognitive decline group (Fig. 2a) showed strong links between various cognitive domains and daily functions ( $r$ ranging from 0.47 to 0.63 ). For example, the range of impaired functions, such as subjective cognition, walking, getting along with other people, housekeeping, and total function, were negatively correlated with attention and processing speed. Executive function domain also had negative correlation with subjective cognition, walking, self-care, housekeeping and total function impairment in this subgroup. Housekeeping dysfunction, which is another aspect, was the most sensitive function related to diverse cognitive domains including composite BAC-A score, verbal memory, attention and processing speed, executive function, and emotional inhibition. In addition, walk dysfunction was correlated with motor speed, attention and processing speed and executive function; subjective cognition dysfunction was also correlated with BAC-A composite score, attention and processing speed, executive function and emotional inhibition. After adjusting for the effect of age to obtain partial correlations, significant correlations were still noted between subjective cognition and BAC-A composite score, subjective walking function and motor speed, subjective housekeeping function and measurements of verbal memory, processing speed, and executive function (not shown in Fig. 2). In the subgroup without cognitive decline (Fig. 2b), no correlation was found between functional and cognitive domains. Thus, the different correlation patterns of the two subgroups revealed heterogeneous effects on daily function by the longitudinal cognitive profile.

\section{Discussion}

To the best of our knowledge, this longitudinal study is the first to provide a comprehensive picture of BD patients with cognitive decline through long-term follow-up, and the results suggested the existence of subgroups with different cognitive trajectories. We classified our patients into two groups according to presence or absence of cognitive decline and correlated cognitive decline to their daily dysfunctions. We revealed that old age, baseline $\mathrm{BMI}>25$, and more number of manic episodes are risk factors for cognitive decline. Furthermore, we demonstrated a hinge at the age of 40 years with steeper decline of cognition in the cognitive decline group.

In our study, the picture of BD patients with longitudinal cognitive decline shows substantial degree of impairment, rather than on only some specific cognitive domains, which is consistent with most previous cross-sectional studies (Van Rheenen et al. 2020). Moreover, the magnitude of cognitive dysfunction reported in previous cross-sectional studies, which used BAC-A to assess cognitive function in BD, is similar to that obtained by combining the two subgroups in the present study (Bauer et al. 2015, Terachi et al. 2017). Our findings, showing longitudinal changes of BD are robust as suggested by those of 
previous cross-sectional literature. On the other hand, our findings of longitudinal cognitive heterogeneity in a subset of patients with BD are important and can partially explain the diverse and inconsistent findings for clinical characteristics and risk factor identification in the literature. In our study, the longitudinal cognitive decline group comprised nearly one-third of our sample, and this group may be established as a specific subtype of BD. Such longitudinal cognitive profiles can potentially facilitate further genetic and biological studies and help clinicians develop more effective intervention strategies (Solé et al. 2017). As we had suggested, the heterogeneity of the cognitive trajectory conformed to the progress of illness in clinical manifestation staging models including cognitive deterioration (de la Fuente-Tomas et al. 2020). Our findings revealed that cognitive decline varies from one patient to another, with decline in certain patients while others remain stable. Conversely, we may say that some patients maintained relatively stable cognitive function might be cognitive reserve, which reflects the partial capacity of the brain to endure neuropathology and minimize clinical cognitive deficits (Kohler et al. 2019). For the statement about the paradigms of cognitive impairment in BD as persistently stable or progressive are not exclusive or may partially overlap (Allott et al. 2020). The verification of heterogeneity of longitudinal cognitive change in our BD samples further comprehend the different faces of cognition in BD.

The risk factors identified for cognitive decline in BD suggest the converging evidence that patients with BD show cognitive impairment related to the clinical course and inflammation theory. For example, a cohort study that assessed longitudinal cognitive changes in BD showed that a higher number of manic episodes is associated with a decrease in global cognition as well as working memory and visual memory (Sanchez-Morla et al. 2019). However, most previous studies have not considered potential heterogeneity and have thus compared all BD together with healthy controls, leading to masking subgroup findings of cognitive change over time in BD (Santos et al. 2014, Ryan et al. 2016, Schouws et al. 2016, Sanchez-Morla et al. 2019). Previous studies have also implied the association of cognitive impairment with other adverse clinical characteristics, such as psychotic features or duration of illness; however, this link was not confirmed in other reports and neither in our study (Suwalska et al. 2001, Bora et al. 2007, Volkert et al. 2016). Moreover, current evidence associates cognitive deficits observed in individuals diagnosed with BD with an increased inflammatory state and decreased neurotrophic factors (Bauer et al. 2014). Recently, obesity has been proven to be associated with chronic systemic inflammation and the release of proinflammatory cytokines (Rodriguez-Hernandez et al. 2013). Peterman et al. also suggested the combined negative effects of obesity and diagnosis of BD on cognition (Peterman et al. 2020). Although our finding implied the pathway from being overweight or obese to cognitive decline in patients with $\mathrm{BD}$, very little is known about why some patients with $\mathrm{BD}$ develop significant cognitive decline while others remain cognitively intact. We recommend that interventions targeting the risk factors of manic episode and high BMI would be effective in preventing cognitive decline in patients with $\mathrm{BD}$ and for detecting the underlying mechanism. Moreover, the subgroup of $\mathrm{BD}$ patients with cognitive decline showed steeper changes after the age of 40 years compared with those without cognitive decline, which is consistent with decline associated with accelerated aging in executive functions in BD (Seelye et al. 2019). We suggested that clinicians should pay attention to patients' 
cognitive function before they reach middle age. Future research should focus on early intervention to determine whether it can delay the decline of cognition in BD.

Psychosocial function is a person's ability to perform activities of daily living and to be involved in meaningful interpersonal relationships. As per our results, patients with BD have difficulties in several areas of function during their remitted status. These results are consistent with those of other studies demonstrating that most patients with BD have functional difficulties (Rosa et al. 2009, Samalin et al. 2016, Comes et al. 2017). However, there was no difference of functional outcome in patients with and without cognitive decline assessed by WHODAS 2.0. There may be possible floor effect of this subjective assessment for this population. We used the WHODAS 2.0 for evaluating our patients, rather than disease-specific questionnaires, such as Functioning Assessment Short Test (FAST), because the former disability assessment is more informative by comparing with general norm or other diseases. Results from the partial correlation analysis between cognitive and functional domains, controlling for age, indicated that the cognitive profile was correlated with some functional domains mainly in the subgroup of $\mathrm{BD}$ patients with cognitive decline. These results are in line with those of cross-sectional studies showing that the cognition-function relationship may be weaker among patients without cognitive deficits than among those with cognitive impairment (Moore et al. 2015). The distinct correlation results among patients with BD also responded to those of a previous study conducted by Sole et al. They suggested the more robust correlation between the poor function group of BD to their cognitive function and smaller correlations in less functional impair BD (Sole et al. 2018). In addition, the cluster of poor functional BD classified by Sole et al. revealed that the occupational domain was associated with executive function and verbal learning tests, which is consistent with our findings even with different cognitive measurements in the current study (Sole et al. 2018).

Several limitations should be considered of this study. First, our study sample was recruited from a tertiary psychiatry hospital where patients have more severe degree of illnesses. This is a potentially biased sample and may limit the generalizability of our findings to the whole BD population, especially the proportion with cognitive decline. Second, without a healthy control group, cognitive decline in patients should be viewed as evidence of relative cognitive decline. Furthermore, the restricted sample size limited the power to examine interaction effects among potential variables, such as interaction of disease course and medications. Third, WHODAS 2.0 is an interviewer-administered tool assessing subjective disability; a combination of objective measures may provide a more comprehensive outcome picture for BD patients with and without cognitive decline. Four, underlying mechanism between risk factors, cognitive decline, and functioning stilled need further investigation. Finally, the dichotomous categorized definition about cognitive decline in our study is arbitrary. However, the potential of misclassification is non-differential and bias the odds ratio toward the null, which suggested the robustness of our findings.

\section{Conclusion}


Our results specify and characterize cognitive heterogeneity in BD longitudinally, which may facilitate further genetic and biological studies to define more valid BD subtypes. We revealed risk factors for cognitive decline and suggested aggressive treatment to prevent mania relapse and control body weight. The heterogeneity of cognitive decline in BD should be considered, thus more individualized intervention for patients with BD could be investigated in future research.

\section{Declarations}

\section{Ethics approval and consent to participate:}

All the participants have informed consent. The Research Ethics Committee of Taipei City Hospital approved our study (TCHIRB-1-703103).

\section{Consent for publication:}

Not applicable

\section{Availability of data and material:}

Restrictions apply to the availability of the data that support the findings of this study, which were guarded by IRB regulations for data access in the recruitment sites. Part of the anonymous data maybe available upon request from the corresponding author ( $\mathrm{PH}$. Kuo).

\section{Competing interests:}

The authors declare that they have no competing interests.

\section{Funding:}

This work was supported by the Ministry of Science and Technology (MOST 107-2314-B-532-001; 1052628-B-002-028-MY3) and National Health Research Institutes (NHRI-EX106-10627NI). The funders played no role in the study design, collection, analysis or interpretation of the data, writing the manuscript, or the decision to submit the paper for publication.

\section{Authors' contributions:}

WYC performed conceptualization, funding acquisition, project administration and writing the original draft. MCH, SKL, HCL, PYC and CCC performed investigation and resources. YCL and CEC done software analysis and data curation. CJK performed the suggestion for investigation, resources and data curation. 
PHK is major for supervision, methodology and reviewing the manuscript. All authors read and approved the final manuscript.

\section{Acknowledgments:}

We thank the Center for Public Health, Department of Education and Research, Taipei City Hospital, Taiwan, for their valuable contributions in data management and statistical analysis.

\section{References}

1. Allott, K. and T. E. Van Rheenen. The complexities of understanding cognitive trajectory in bipolar disorder. Bipolar Disord. 2020.

2. Atre-Vaidya, N., M. A. Taylor, M. Seidenberg, R. Reed, A. Perrine and F. Glick-Oberwise. Cognitive deficits, psychopathology, and psychosocial functioning in bipolar mood disorder. Neuropsychiatry Neuropsychol Behav Neurol. 1998;11(3): 120-126.

3. Bauer, I. E., R. S. Keefe, M. Sanches, R. Suchting, C. E. Green and J. C. Soares. Evaluation of cognitive function in bipolar disorder using the Brief Assessment of Cognition in Affective Disorders (BAC-A). J Psychiatr Res. 2015;60: 81-86.

4. Bauer, I. E., M. C. Pascoe, B. Wollenhaupt-Aguiar, F. Kapczinski and J. C. Soares. Inflammatory mediators of cognitive impairment in bipolar disorder. J Psychiatr Res. 2014;56: 18-27.

5. Bora, E. Developmental trajectory of cognitive impairment in bipolar disorder: comparison with schizophrenia. Eur Neuropsychopharmacol. 2015;25(2): 158-168.

6. Bora, E., C. Hidiroglu, A. Ozerdem, O. F. Kacar, G. Sarisoy, F. Civil Arslan, et al. Executive dysfunction and cognitive subgroups in a large sample of euthymic patients with bipolar disorder. Eur Neuropsychopharmacol. 2016;26(8): 1338-1347.

7. Bora, E. and C. Pantelis. Meta-analysis of Cognitive Impairment in First-Episode Bipolar Disorder: Comparison With First-Episode Schizophrenia and Healthy Controls. Schizophr Bull. 2015;41(5): 1095-1104.

8. Bora, E., S. Vahip, F. Akdeniz, A. S. Gonul, A. Eryavuz, M. Ogut, et al. The effect of previous psychotic mood episodes on cognitive impairment in euthymic bipolar patients. Bipolar Disord. 2007;9(5): 468477.

9. Bourne, C., O. Aydemir, V. Balanza-Martinez, E. Bora, S. Brissos, J. T. Cavanagh, et al. Neuropsychological testing of cognitive impairment in euthymic bipolar disorder: an individual patient data meta-analysis. Acta Psychiatr Scand. 2013;128(3): 149-162.

10. Chen, R. A., C. Y. Lee, Y. Lee, C. F. Hung, Y. C. Huang, P. Y. Lin, et al. Defining cognitive profiles of depressive patients using the Brief Assessment of Cognition in Affective Disorders. PeerJ. 2019;7: e7432. 
11. Cholet, J., A. Sauvaget, J. M. Vanelle, C. Hommet, K. Mondon, J. P. Mamet, et al. Using the Brief Assessment of Cognition in Schizophrenia (BACS) to assess cognitive impairment in older patients with schizophrenia and bipolar disorder. Bipolar Disord. 2014;16(3): 326-336.

12. Cipriani, G., S. Danti, C. Carlesi, D. M. Cammisuli and M. Di Fiorino. Bipolar Disorder and Cognitive Dysfunction: A Complex Link. J Nerv Ment Dis. 2017;205(10): 743-756.

13. Comes, M., A. Rosa, M. Reinares, C. Torrent and E. Vieta. Functional Impairment in Older Adults With Bipolar Disorder. J Nerv Ment Dis. 2017;205(6): 443-447.

14. de la Fuente-Tomas, L., P. Sierra, M. Sanchez-Autet, B. Arranz, A. Garcia-Blanco, G. Safont, et al. A clinical staging model for bipolar disorder: longitudinal approach. Transl Psychiatry. 2020;10(1): 45.

15. Diniz, B. S., A. L. Teixeira, F. Cao, A. Gildengers, J. C. Soares, M. A. Butters, et al. History of Bipolar Disorder and the Risk of Dementia: A Systematic Review and Meta-Analysis. Am J Geriatr Psychiatry. 2017;25(4): 357-362.

16. Duarte, W., R. Becerra and K. Cruise. The Relationship Between Neurocognitive Functioning and Occupational Functioning in Bipolar Disorder: A Literature Review. Eur J Psychol. 2016;12(4): 659678.

17. Gildengers, A. G., D. Chisholm, M. A. Butters, S. J. Anderson, A. Begley, M. Holm, et al. Two-year course of cognitive function and instrumental activities of daily living in older adults with bipolar disorder: evidence for neuroprogression? Psychol Med. 2013;43(4): 801-811.

18. Gildengers, A. G., B. H. Mulsant, A. Begley, S. Mazumdar, A. V. Hyams, C. F. Reynolds lii, et al. The longitudinal course of cognition in older adults with bipolar disorder. Bipolar Disord. 2009;11(7): 744752.

19. Goldberg, J. F., M. Harrow and L. S. Grossman. Course and outcome in bipolar affective disorder: a longitudinal follow-up study. Am J Psychiatry. 1995;152(3): 379-384.

20. Kohler, S. and E. Marlinge. Cognitive aging of bipolar patients. Geriatr Psychol Neuropsychiatr Vieil. 2019;17(2): 179-188.

21. Lee, C. Y., L. J. Wang, Y. Lee, C. F. Hung, Y. C. Huang, M. I. Lee, et al. Differentiating bipolar disorders from unipolar depression by applying the Brief Assessment of Cognition in Affective Disorders. Psychol Med. 2018;48(6): 929-938.

22. Martino, D. J., S. A. Strejilevich, M. Scapola, A. Igoa, E. Marengo, E. D. Ais, et al. Heterogeneity in cognitive functioning among patients with bipolar disorder. J Affect Disord. 2008;109(1-2): 149-156.

23. Milborrow, S. (2011). Earth Package in R.

24. Moore, R. C., A. L. Harmell, P. D. Harvey, C. R. Bowie, C. A. Depp, A. E. Pulver, et al. Improving the understanding of the link between cognition and functional capacity in schizophrenia and bipolar disorder. Schizophr Res. 2015;169(1-3): 121-127.

25. Moorhead, T. W., J. McKirdy, J. E. Sussmann, J. Hall, S. M. Lawrie, E. C. Johnstone, et al. Progressive gray matter loss in patients with bipolar disorder. Biol Psychiatry. 2007;62(8): 894-900. 
26. Mur, M., M. J. Portella, A. Martinez-Aran, J. Pifarre and E. Vieta. Long-term stability of cognitive impairment in bipolar disorder: a 2-year follow-up study of lithium-treated euthymic bipolar patients. J Clin Psychiatry. 2008;69(5): 712-719.

27. Peterman, J. S., D. F. Marshall, E. Lamping, R. E. Easter, P. Babu, S. A. Langenecker, et al. Decreased working memory capacity among individuals with a mood disorder who have increased metabolic burden. J Affect Disord. 2020;266: 387-393.

28. Robinson, L. J. and I. N. Ferrier. Evolution of cognitive impairment in bipolar disorder: a systematic review of cross-sectional evidence. Bipolar Disord. 2006;8(2): 103-116.

29. Rodriguez-Hernandez, H., L. E. Simental-Mendia, G. Rodriguez-Ramirez and M. A. Reyes-Romero. Obesity and inflammation: epidemiology, risk factors, and markers of inflammation. Int $\mathrm{J}$ Endocrinol. 2013;2013: 678159.

30. Rosa, A. R., M. Reinares, C. Franco, M. Comes, C. Torrent, J. Sanchez-Moreno, et al. Clinical predictors of functional outcome of bipolar patients in remission. Bipolar Disord. 2009;11(4): 401-409.

31. Ryan, K. A., S. Assari, B. D. Pester, K. Hinrichs, K. Angers, A. Baker, et al. Similar Trajectory of Executive Functioning Performance over 5 years among individuals with Bipolar Disorder and Unaffected Controls using Latent Growth Modeling. J Affect Disord. 2016;199: 87-94.

32. Samalin, L., I. de Chazeron, E. Vieta, F. Bellivier and P. M. Llorca. Residual symptoms and specific functional impairments in euthymic patients with bipolar disorder. Bipolar Disord. 2016;18(2): 164173.

33. Samame, C., D. J. Martino and S. A. Strejilevich. Longitudinal course of cognitive deficits in bipolar disorder: a meta-analytic study. J Affect Disord. 2014;164: 130-138.

34. Sanchez-Morla, E. M., A. Lopez-Villarreal, E. Jimenez-Lopez, A. I. Aparicio, V. Martinez-Vizcaino, R. J. Roberto, et al. Impact of number of episodes on neurocognitive trajectory in bipolar disorder patients: a 5-year follow-up study. Psychol Med. 2019;49(8): 1299-1307.

35. Santos, J. L., A. Aparicio, A. Bagney, E. M. Sanchez-Morla, R. Rodriguez-Jimenez, J. Mateo, et al. A five-year follow-up study of neurocognitive functioning in bipolar disorder. Bipolar Disord. 2014;16(7): 722-731.

36. Schouws, S. N., H. C. Comijs, A. Dols, A. T. Beekman and M. L. Stek. Five-year follow-up of cognitive impairment in older adults with bipolar disorder. Bipolar Disord. 2016;18(2): 148-154.

37. Seelye, A., P. Thuras, B. Doane, C. Clason, W. VanVoorst and S. Urosevic. Steeper aging-related declines in cognitive control processes among adults with bipolar disorders. J Affect Disord. 2019;246: 595-602.

38. Solé, B., E. Jiménez, C. Torrent, M. Reinares, C. D. M. Bonnin, I. Torres, et al. Cognitive Impairment in Bipolar Disorder: Treatment and Prevention Strategies. Int J Neuropsychopharmacol. 2017;20(8): 670-680.

39. Sole, B., C. M. Bonnin, E. Jimenez, C. Torrent, I. Torres, C. Varo, et al. Heterogeneity of functional outcomes in patients with bipolar disorder: a cluster-analytic approach. Acta Psychiatr Scand. 2018;137(6): 516-527. 
40. Suwalska, A., A. Borkowska and J. Rybakowski. [Cognitive deficits in the bipolar affective disorder]. Psychiatr Pol. 2001;35(4): 657-668.

41. Szmulewicz, A., M. P. Valerio and D. J. Martino. Longitudinal analysis of cognitive performances in recent-onset and late-life Bipolar Disorder: A systematic review and meta-analysis. Bipolar Disord. 2020;22(1): 28-37.

42. Terachi, S., T. Yamada, S. Pu, K. Yokoyama, H. Matsumura and K. Kaneko. Comparison of neurocognitive function in major depressive disorder, bipolar disorder, and schizophrenia in later life: A cross-sectional study of euthymic or remitted, non-demented patients using the Japanese version of the Brief Assessment of Cognition in Schizophrenia (BACS-J). Psychiatry Res. 2017;254: 205-210.

43. Torrent, C., A. Martinez-Aran, C. del Mar Bonnin, M. Reinares, C. Daban, B. Sole, et al. Long-term outcome of cognitive impairment in bipolar disorder. J Clin Psychiatry. 2012;73(7): e899-905.

44. Ustun, T. B., N. Kostanjesek, S. Chatterji, J. Rehm and O. World Health (2010). Measuring health and disability : manual for WHO Disability Assessment Schedule (WHODAS 2.0) / edited by T.B. Üstün, N. Kostanjsek, S. Chatterji, J.Rehm. Geneva, World Health Organization.

45. Van Rheenen, T. E., K. E. Lewandowski, I. E. Bauer, F. Kapczinski, K. Miskowiak, K. E. Burdick, et al. Current understandings of the trajectory and emerging correlates of cognitive impairment in bipolar disorder: An overview of evidence. Bipolar Disord. 2020;22(1): 13-27.

46. Vanegas, J. and F. Vasquez. [Multivariate Adaptive Regression Splines (MARS), an alternative for the analysis of time series]. Gac Sanit. 2017;31(3): 235-237.

47. Volkert, J., M. A. Schiele, J. Kazmaier, F. Glaser, K. C. Zierhut, J. Kopf, et al. Cognitive deficits in bipolar disorder: from acute episode to remission. Eur Arch Psychiatry Clin Neurosci. 2016;266(3): 225-237.

\section{Figures}




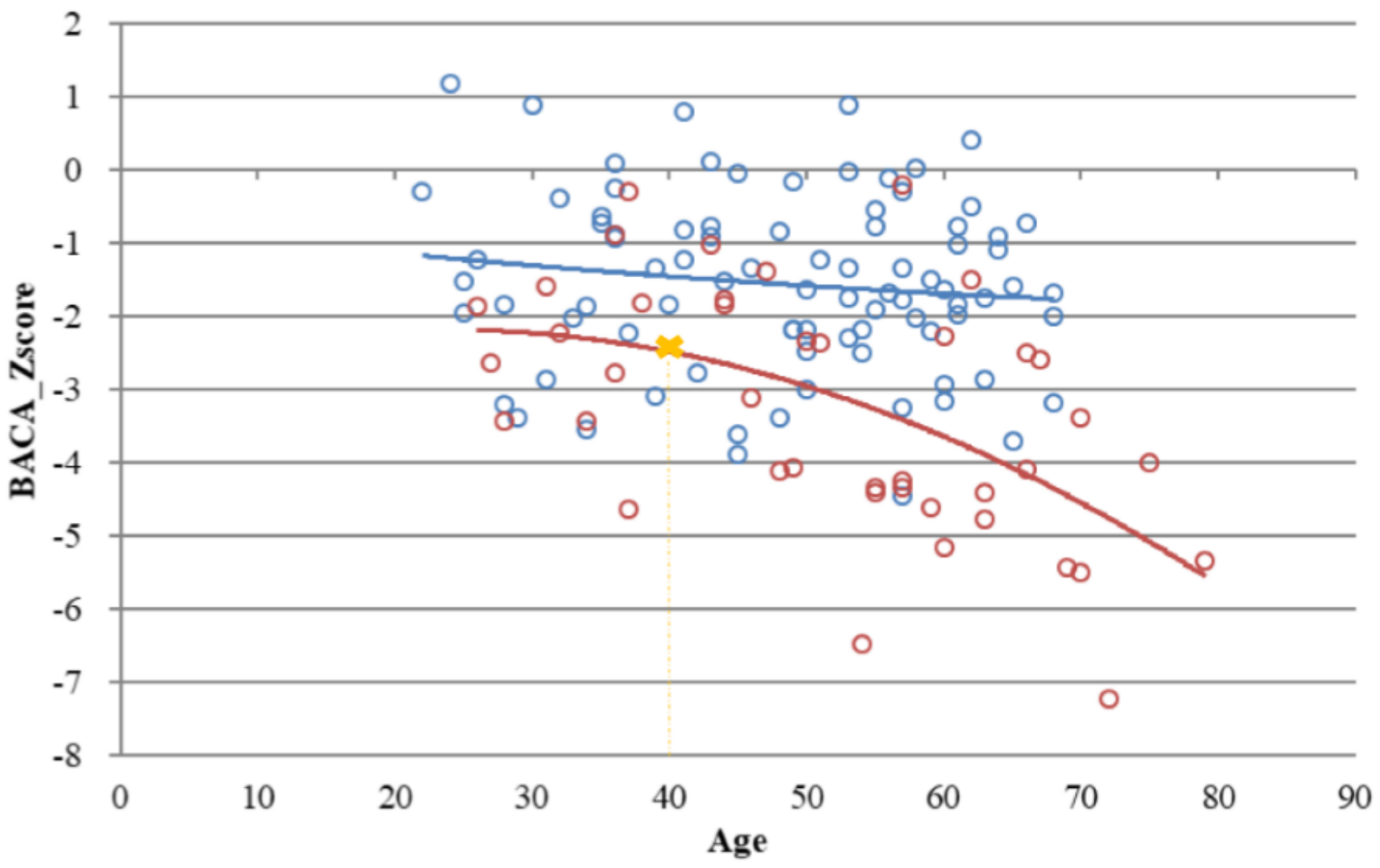

Without cognitive decline $\quad$ With cognitive decline

\section{Figure 1}

Scatterplot between age and BAC-A score in BD patients with or without cognitive decline. The figure depicts steeper decrease in BAC-A Z score with hinge points (yellow mark) at the age of 40 years in BD patients with cognitive decline. The slope of red curve showed -0.1009 SD per year after age 40 .

a

Patients with cognitive decline

\begin{tabular}{|c|c|c|c|c|c|c|c|c|c|}
\hline-0.4 & $-0.6^{*}$ & -0.41 & -0.3 & -0.43 & $-0.56^{*}$ & -0.18 & -0.07 & BAC-A Composite Score & 0.4 \\
\hline-0.15 & -0.27 & -0.36 & -0.11 & -0.27 . & $-0.47^{*}$ & -0.08 & -0.02 & Verbal Memory & 0.2 \\
\hline 0.2 & -0.12 & $-0.5^{\circ}$ & 0.24 & -0.29 & 0.26 & -0.03 & 0.07 & Motor Speed & 0 \\
\hline 0 & -0.34 & -0.07 & 0.08 & -0.15 & -0.16 & -0.40 & 0.24 & Working Memory & -0.2 \\
\hline-0.2 & -0.22 & -0.44 & -0.14 & -0.06 & -0.33 & 0.06 & -0.12 & Verbal Fluency & -0.4 \\
\hline$-0.51^{*}$ & $-0.51^{*}$ & $-0.5^{*}$ & -0.35 & $-0.54^{*}$ & $-0.55^{*}$ & -0.07 & -0.19 & Attention and Processing Speed & -0.6 \\
\hline$-0.51^{*}$ & $-0.6^{*}$ & $-0.47^{*}$ & $-0.51^{*}$ & -0.3 & $-0.63^{*}$ & -0.01 & -0.13 & Executive Function & \\
\hline 0.36 & $0.53^{\circ}$ & 0.3 & 0.17 & 0.43 & $0.5^{*}$ & 0.25 & -0.04 & Emotion Inhibition Index & \\
\hline 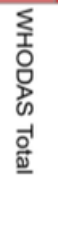 & 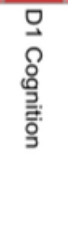 & 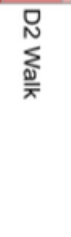 & 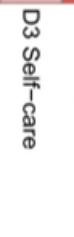 & 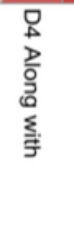 & 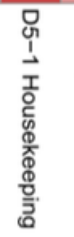 & 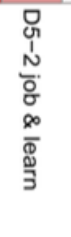 & 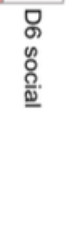 & & \\
\hline
\end{tabular}

b

\section{Patients without cognitive decline}

\begin{tabular}{|c|c|c|c|c|c|c|c|c|}
\hline-0.18 & -0.2 & -0.13 & -0.06 & -0.31 & -0.16 & -0.17 & 0 & BAC-A Composite Score \\
\hline-0.12 & -0.13 & -0.11 & -0.11 & -0.16 & -0.11 & -0.3 & 0.04 & Verbal Memory \\
\hline-0.29 & -0.17 & -0.22 & -0.07 & -0.29 & -0.15 & -0.28 & -0.15 & Motor Speed \\
\hline-0.17 & -0.27 & -0.08 & -0.14 & -0.33 & -0.29 & -0.01 & -0.06 & Working Memory \\
\hline-0.03 & -0.06 & -0.03 & -0.18 & -0.06 & -0.21 & 0.01 & 0.07 & Verbal Fluency \\
\hline-0.15 & -0.12 & -0.04 & 0.11 & -0.27 & -0.12 & -0.07 & 0.03 & Attention and Processing Speed \\
\hline-0.05 & -0.12 & -0.16 & -0.26 & -0.15 & -0.11 & 0.22 & -0.14 & Executive Function \\
\hline 0.13 & 0.03 & 0.19 & -0.2 & 0.28 & 0.28 & 0.1 & -0.16 & Emotion Inhibition Index \\
\hline 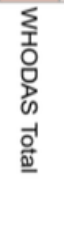 & 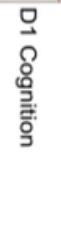 & 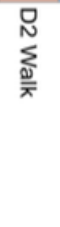 & 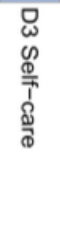 & 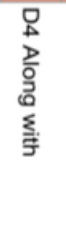 & 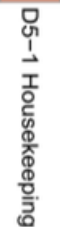 & 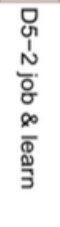 & 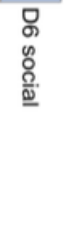 & \\
\hline
\end{tabular}

Figure 2 
Correlations between cognitive and functional domains in BD. ${ }^{\star} p<0.05$ 